\title{
Führt Cytisin langfristiger zur Abstinenz?
}

Das pflanzliche Alkaloid Cytisin ist wie Vareniclin ein

Partialagonist der nikotinischen Acetylcholinrezeptoren.

Es wurde früher v.a. in osteuropäischen Ländern zur

Tabakentwöhnung eingesetzt und führt im Vergleich zu

Placebo zu einer besseren Kurz- und Langzeitabstinenz.

In einer neuseeländischen Studie sollte das Alkoloid zeigen, ob es einer Nikotinersatztherapie zumindest nicht unterlegen ist. N Engl J Med 2014; 371: 2353-2362

Die Teilnehmer dieses offenen, randomisierten, kontrollierten Parallelgruppenvergleichs wurden über die nationale Raucher-Hotline rekrutiert. In die Studie konnten Erwachsene ab 18 Jahren mit einem täglichen Zigarettenkonsum aufgenommen werden. Insgesamt 1310 Personen, die mit dem Wunsch zur Rauchabstinenz bei der Raucher-Hotline Neuseelands anriefen, wurden zufällig 2 Gruppen zugeordnet: 655 Teilnehmer erhielten Cytisin-Tabletten für 25 Tage Anwendung per Kurier und weitere 655 konnten sich mit Apothekengutscheinen Nikotinpflaster, -kaugummis und/oder -lutschtabletten besorgen, die für einen Zeitraum von 8 Wochen ausreichen würden. In der Cytisin-Gruppe sollte das Rauchen nach eigener Maßgabe innerhalb der ersten 4 Tage reduziert werden, sodass am 5. Tag eine absolute Rauchkarenz herrschte (Quit Date). Die eingenommene Zahl an Cytisin-Tabletten sollte schrittweise von anfänglich max. 6 auf 2 pro Tag reduziert werden. Alle 566 Männer und 744 Frauen (Durchschnittsalter 38 Jahre) erhielten eine telefonische Verhaltensberatungen von durchschnittlich 3 Gesprächen à 10-15 min über 8 Wochen. Den primären Endpunkt bildete die anhaltende Rauchabstinenz 1 Monat nach dem Quit Date. Zusätzlich sollten die CytisinAnwender nach einer Woche und nach einem Monat Auskunft geben, ob sie das Mittel als Entwöhnungshilfe empfehlen würden.

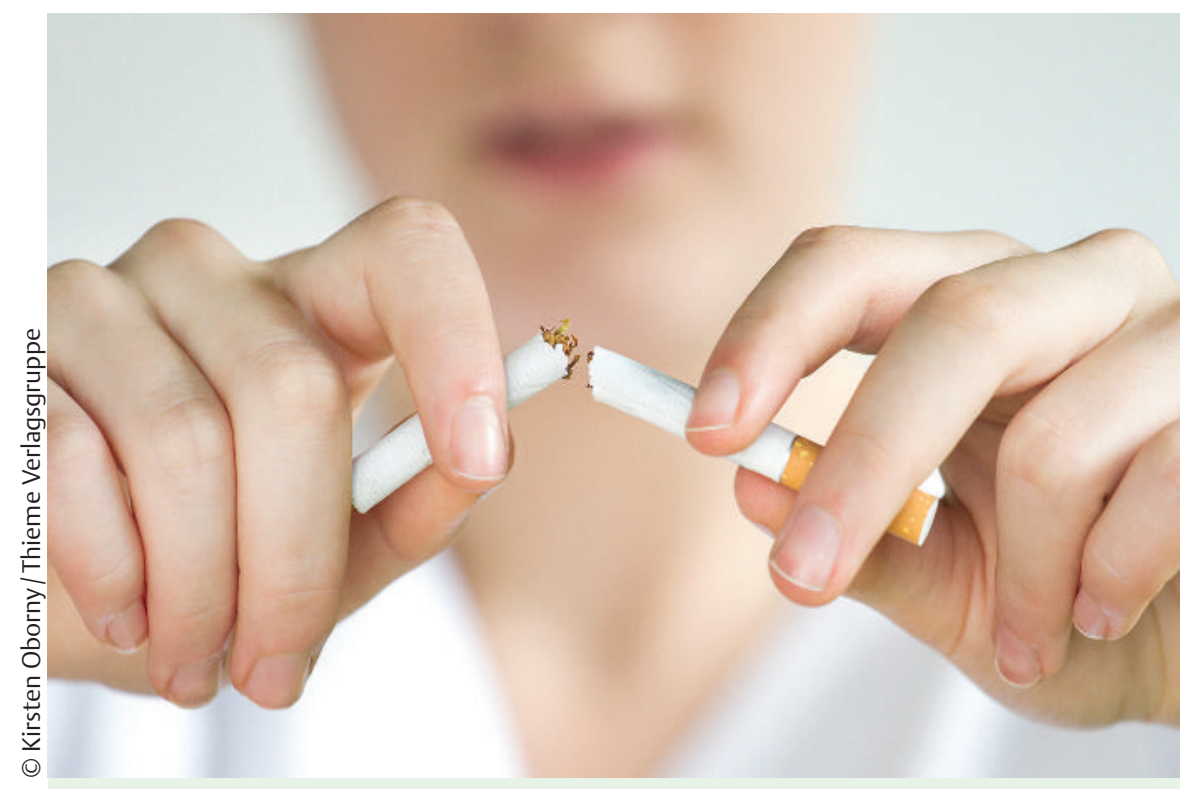

Menschen, die mit dem Rauchen aufhören möchten, stehen eine Vielzahl unterschiedlicher Beratungsangebote und Entwöhnungshilfen zur Verfügung. Doch welcher Weg führt langfristig zur Abstinenz?
Frauen erfolgreicher abstinent

$\nabla$

Gegenüber der Nikotinersatztherapie lag die Abstinenzrate in der Cytisin-Gruppe mit 40 vs. $31 \%$ deutlich höher (Unterschied 9,3 Prozentpunkte). Die Number needed to Treat betrug 11 und die Odds Ratio für Abstinenz unter Cytisin 1,5 $(\mathrm{p}=0,003)$. Die statistisch signifikante Überlegenheit von Cytisin nach einem Monat zeigte sich nur bei Frauen, bei Männern erreicht sie nicht das Signifikanzniveau (Nichtunterlegenheit gegenüber Nikotinersatztherapie). In der Cytisin-Gruppe befolgten $53 \%$ und unter Nikotinersatztherapie $67 \%$ die Therapievorgaben. Einen Monat nach dem Quit Date nahmen $22 \%$ der Cytisin-Anwender und 55\% der Teilnehmer aus der Vergleichsgruppe weiterhin die Entwöhnungshilfen ein.

Insgesamt rauchten zum Quit Date unter Cytisin 21\% und unter Nikotinersatztherapie 34\% der Teilnehmer. Die mediane Zeit bis zum Rückfall nach der Rauchkarenz ab dem 5. Tag lag in der Cytisin-Gruppe bei 53 Tagen und mit Nikotinersatzmitteln bei 11 Tagen (Hazard Ratio: 0,8, $\mathrm{p}=0,001)$. Die Nebenwirkungen waren überwiegend leicht oder moderat und traten unter Cytisin häufiger auf (v.a. Übelkeit, Erbrechen, Schlafstörungen, $\mathrm{p}<0,001)$. Unter den Cytisin-Anwendern mit Nebenwirkungen gaben 1 Woche nach dem Quit Date $89 \%$ und 1 Monat danach $82 \%$ an, dass sie Cytisin zur Rauchentwöhnung empfehlen würden.

\section{Fazit}

Kombiniert mit kurzen telefonischen Verhaltensberatungen war Cytisin in dieser Studie der Nikotinersatztherapie bei dem Ziel einer anhaltenden Rauchabstinenz 1 Monat nach einer Rauchkarenz überlegen. Cytisin erhöhte die Rate leichter bis moderater Nebenwirkungen. Die Autoren halten einen direkten Vergleich mit Vareniclin für gerechtfertigt.

Matthias Manych, Berlin 